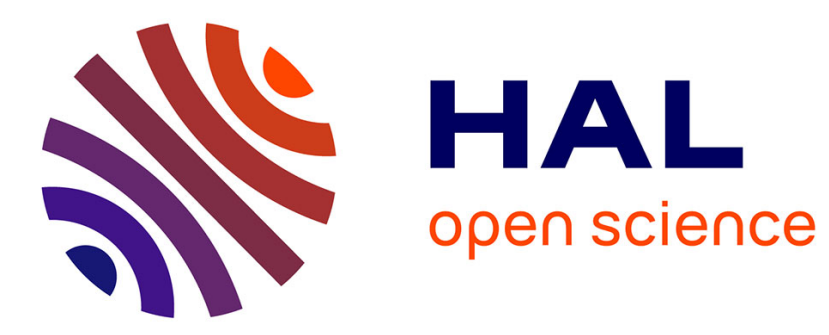

\title{
Hydrodynamic instability and flow reduction in polymer brush coated channels
}

Sofia Biagi, Lorenzo Rovigatti, Mehdi Abbasi, Lionel Bureau, Francesco

Sciortino, Chaouqi Misbah

\section{- To cite this version:}

Sofia Biagi, Lorenzo Rovigatti, Mehdi Abbasi, Lionel Bureau, Francesco Sciortino, et al.. Hydrodynamic instability and flow reduction in polymer brush coated channels. Soft Matter, 2021, 17 (40), pp.9235-9245. 10.1039/d1sm00638j . hal-03450373

\section{HAL Id: hal-03450373 \\ https://hal.science/hal-03450373}

Submitted on 26 Nov 2021

HAL is a multi-disciplinary open access archive for the deposit and dissemination of scientific research documents, whether they are published or not. The documents may come from teaching and research institutions in France or abroad, or from public or private research centers.
L'archive ouverte pluridisciplinaire HAL, est destinée au dépôt et à la diffusion de documents scientifiques de niveau recherche, publiés ou non, émanant des établissements d'enseignement et de recherche français ou étrangers, des laboratoires publics ou privés. 


\title{
Hydrodynamic instability and flow reduction in polymer brush coated channels
}

\author{
Sofia Biagi, ${ }^{1,2, *}$ Lorenzo Rovigatti, ${ }^{2, \dagger}$ Mehdi Abbasi, ${ }^{1, \ddagger}$ Lionel \\ Bureau, ${ }^{1, \S}$ Francesco Sciortino, ${ }^{2,3,}$ ๆ and Chaouqi Misbah ${ }^{1, * *}$ \\ ${ }^{1}$ Université Grenoble /CNRS, LIPhy UMR 5588, Grenoble, F-38401, France \\ ${ }^{2}$ Dipartimento di Fisica, Sapienza-Universitá di Roma, Piazzale A. Moro 5, 00185 Roma, Italy \\ ${ }^{3}$ Istituto Sistemi Complessi (ISC), Via dei Taurini 19, 00185 Roma, Italy
}

(Dated: July 26, 2021)

\begin{abstract}
A polymer brush is a passive medium. At equilibrium the knowledge of its chemical composition and thickness is enough for a full system characterization. However, when the brush is exposed to fluid flow it reveals a much more intriguing nature, displaying intermediate properties between those typically found in solids, porous materials and active-matter systems. Here we investigate such a rich behavior via numerical simulations. We focus on the brush hydrodynamic response at low Reynolds numbers, observing a significant fluid flow reduction inside a polymer-brush coated channel. We find that the reduction of the flow inside the channel is significantly larger than what would happen if the brush effect consisted only in reducing the effective channel width. This amplified reduction is understood as being due to a morphological instability of the brush-liquid interface which is shown to have an elastic origin: the mechanical stress acting on the brush due to the imposed flow is partially released by the interface modulation. In turn, this modulation dissipates in the surrounding fluid more energy than a flat interface, causing a reduction of flow velocity. Our results and interpretations provide an explanation for recent experimental measurements.
\end{abstract}

PACS numbers: ???????

\section{INTRODUCTION}

Polymer brushes are three-dimensional matrices of densely grafted polymers, providing filamentous surface coatings. From the synthetic point of view they can be manipulated under many respects (e.g. polymer species, surface geometry, grafting architecture) in order to finely tune the physico-chemical properties of a desired surface (the grafted substrate) [1-4]; however, they also constitute the puzzling ingredient of many mammalian organs [5-7]. In the first case, experimentalists exploit the brush equilibrium features and the versatility of the polymerization process to control its responsivenesses at different electro-chemical stimuli. In the second case, since many of the polymer-decorated organs are concerned with the flowing or draining of biological fluids, the research is centered on non-equilibrium features, with the aim to reveal polymer brush dynamics and, consequently, understanding why they have been awarded by Evolution to be the most adequate organ coating.

In order to achieve a complete description of the hydrodynamic response of a polymer brush, one should scrutinize the system by mediating between a continuum point of view, i.e. the possibility to integrate the fluid flow streamlines, and a detailed characterization of the polymer dynamics, zooming in the lengthscales. The majority

\footnotetext{
* sofiabiagi.n@gmail.com

† lorenzo.rovigatti@uniroma1.it

¥ mehdi.abbasi@univ-grenoble-alpes.fr

§ lionel.bureau@univ-grenoble-alpes.fr

I francesco.sciortino@uniroma1.it

** chaouqi.misbah@univ-grenoble-alpes.fr
}

of studies, instead, has focused on only one of those two aspects [8-12]. For instance, a recent work reported on microfluidic experiments in glass capillaries coated with artificial polymer brushes. It has been found that the fluid velocity is reduced significantly more than expected from a channel diameter reduction due to the presence of the brush [12]. Such surprising and non-trivial outcome, however, could not be directly related to a precise brush dynamics, since the experiments lacked resolution at the scale of polymers (the brush thickness ranging in the order of hundred nanometers). Here, we offer a complementary point of view to those experiments: we model the flow in a channel coated by a dense polymer brush with a mesoscale approach, which allows for an investigation of the fluid streamlines together with a detailed description of the actual polymer dynamics. In qualitative agreement with the experiments we find a significant reduction of the flow velocity in the channel, which we relate with the brush features: indeed, we attribute the main origin of this peculiar behaviour to the brush surface deformation. Such a deformation, appearing even at low Reynolds numbers, takes the form of a monochromatic travelling wave, a phenomenon which has been thoroughly characterized in Ref. [13]. In the cited study the wave, experienced for different flow strengths, is detailed in frequencies, wavelengths and oscillation amplitudes and it is also associated to a fluid backward flow measured in the vicinity of the brush interface. Here, in addition, we ascribe the appearance of the surface wave to an instability induced by hydrodynamic shear modes and provide a general condition for its onset. Thus, the present paper develops an analytical study to understand the onset of instability for a polymer brush, treated as an elastic medium. This study supports the numeri- 
cal study based on mesoscale self-consistent simulations, which produce a direct observation of a brush surface modulation under certain conditions. Results and interpretations presented therein offer an explanation for recent microfluidic experiments and allow us to include polymer brushes among those boundaries which induce flow instabilities in a fluid at low Reynolds numbers.

\section{METHODS}

We aim at reproducing the dynamics of a simple Newtonian fluid, modelled explicitly, and of densely grafted polymers. Enclosing in a same numerical simulation both the hydrodynamics and the polymer dynamics requires a model suitable to grasp physics at the mesoscale. We thus resort to coarse-graining methods and opt for the Dissipative Particle Dynamics (DPD) technique [1417]. Indeed, a DPD particle represents not the single molecule, but rather a certain amount of them. Our DPD code applies a uniform coarse-graining both to solvent particles and monomers, actually following the evolution in time of clusters of fluid particles and blobs of monomers. Forces to be integrated at each time step are then adjusted for the zooming-out of lengthscales. A detailed description of the code implementation with the analytical expressions of forces is reported in the Appendix, so, here, we just recall the main features of the method. We avail ourselves of the three standard DPD forces: a conservative one, a dissipative one and a stochastic one. The first one is a soft-core repulsive force, allowing the overlap of clusters: the DPD particle must not have a strict spherical symmetry, due to rearrangement of the real microscopic molecules. The high or low easiness of such rearrangement, meaning the viscous resistance, is represented by the second force, while the third force accounts for the number of collisions among real microscopic molecules. Since all these three DPD forces act between particle pairs and are central in order to enforce local momentum conservation, the correct hydrodynamics at long times is guaranteed. We underline that fluid particles exchange momentum with polymer chains, dragging them. The model is, thus, selfconsistent, accessing both the polymer dynamics, influenced by the imposed flow, and the flow field, perturbed by the presence and motion of the brush.

We show in Fig.1 a sketch of the studied set-up: a parallelepiped box of sides $L_{x}, L_{y}, L_{z}$ containing $N$ coarsegrained DPD particles. A portion of them mimics the simple liquid (not shown), while the rest, $N_{m}$ (in light red), make up the brush monomers. For the sake of ease and computational time we attach polymers only to the bottom wall $z=0$. In the homodisperse case all polymers have the same molecular weight (or polymerization degree): each of the $N_{c h}$ linear polymer chains is composed by $n$ identical monomers so that $N_{m}=n N_{c h}$. Monomers of a same chain are connected by an additional force, derived by a finite extensible nonlinear elastic po- tential (FENE) [18]. The grafting point coordinates are randomly chosen from a uniform distribution and located on bottom surface according to the imposed grafting density $\sigma_{\text {graft }}$, defined as $\sigma_{\text {graft }}=N_{c h} /\left(L_{x} L_{y}\right)$.

We recall that in a slit-pore geometry a laminar flow takes a parabolic shape, as shown in Fig. 1. In order to produce a parabolic flow inside the channel (e.g. along the $x$ direction) a constant acceleration $\vec{A}=A \hat{x}$ is applied to all fluid particles:

$$
m \dot{\vec{v}}_{i}^{\text {fluid }}=\vec{F}_{i j}^{\text {fluid }}+m \vec{A} .
$$

Analytically, this choice is fully equivalent to the application of a pressure drop at the inlet/outlet of the channel and more convenient for simulations where periodic boundary conditions are employed. No-slip at the walls is imposed by applying the bounce-back boundary condition [19]. Different values of $A$ allow us to probe different dynamic regimes, here embodied by the value of the Weissenberg number $W i$, defined as $W i \equiv \frac{t_{\text {brush }}}{t_{\text {flow }}}$, where $t_{\text {brush }}$ is a brush characteristic timescale and $t_{\text {flow }}$ is a timescale introduced by the flow. Later in the text we will introduce a specific definition for the Weissenberg number, which arises naturally when dealing with surface instability.

Physical units are established by comparison with the physical reference system, namely the microfluidic experiments reported in Ref.[12] and the biopolymer brush called endothelial glycocalyx, which covers the inner vessel walls of mammalians [20]. From the ratio of the spacing between different filaments of the glycocalyx network, $d_{\text {glyco }}=20 \mathrm{~nm}[21]$, over the average distance between anchor points of our brush, $d_{\text {graft }}=\sqrt{1 / \sigma_{\text {graft }}}=0.82$ we thus fix the physical lengthscale $l_{\text {phys }}$ :

$$
l_{\text {phys }}=d_{\text {glyco }} / d_{\text {graft }}=24 \cdot 10^{-9} \mathrm{~m} .
$$

The physical mass and time scales, $m_{\text {phys }}$ and $t_{\text {phys }}$, are extracted by the ratio of viscosities and by the ratio of particle energies:

$$
\begin{gathered}
m_{\text {phys }}=\frac{\eta_{\text {phys }}}{\eta_{D P D}} t_{\text {phys }} l_{\text {phys }}, \\
t_{\text {phys }}=\frac{l_{\text {phys }}}{v_{\text {phys }}}=\frac{l_{\text {phys }}}{\sqrt{\frac{3 k_{B} T_{\text {phys }}}{m_{\text {phys }}}}},
\end{gathered}
$$

where $\eta_{\text {phys }}=10^{-3} \mathrm{~Pa} s$ is the viscosity of water at $T_{\text {phys }}=300 \mathrm{~K}, k_{B}$ is the Boltzmann constant and the DPD viscosity $\eta_{D P D}=0.84$ is estimated by a simulation of slit-pore velocity profile in a bare channel, using $\eta=\rho A R^{2} /\left(2 v_{\max }\right)$ with $v_{\max }$ the maximum velocity at $z=R$. Solving the system, we obtain $t_{\text {phys }}=1.8 \cdot 10^{-6} \mathrm{~s}$ and $m_{\text {phys }}=5.1 \cdot 10^{-17} \mathrm{~kg}$. With such choices, the physical values for the brush thickness $h_{b}$ and for the maximum fluid velocity $v_{\max }$ inside the channel are, respectively, of the order of $500 \mathrm{~nm}$ and $1 \mathrm{~cm} / \mathrm{s}$. 


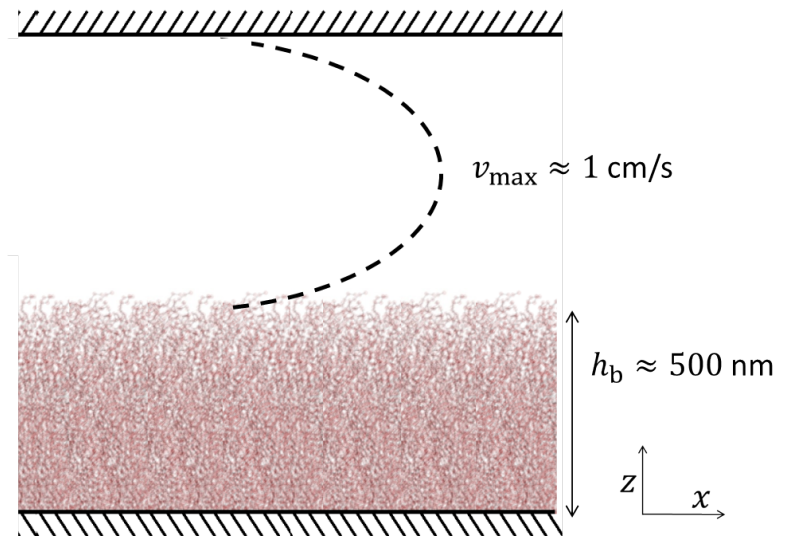

FIG. 1. A sketch of the set-up system, showing the $x z$ section of the slit-pore channel. Two rigid walls are placed at $z=0$ and $z=L_{z}$. The bottom wall is coated by a (homodisperse) polymer brush, whose thickness is named $h_{b}$. The velocity profile inside the channel is parabolic and $v_{\max }$ signals its maximum value.

\section{RESULTS}

At equilibrium the brush conformation results from the balance between configurational entropy, that tends to make chains visit the whole available space, and excluded volume interactions, which disfavors contact between monomers. Theoretical models (mean-field or scaling theories $[22,23]$ ), experiments (especially neutron scattering $[24,25])$ and numerical simulations (Monte Carlo and Molecular Dynamics methods [26, 27]) agree in predicting the monomer distribution, the layer thickness and the way it should scale with the grafting density $\sigma_{\text {graft }}$ and the polymerization degree $n$. Indeed, at equilibrium the brush is properly described by the profile $\rho(z)$, which is the probability distribution of finding a monomer at a distance $z$ from the grafting wall. In Fig. 2 we show $\rho(z)$ for $\sigma_{\text {graft }}=1.5$ and $n=40$ (the brush thickness is $h_{b}=2 \int z \rho(z) d z / \int \rho(z) d z=24.8$ in DPD units [20]) and, in the inset, a comparison between brushes with different grafting densities $\sigma_{\text {graft }}=0.1,0.3,0.5,0.8,1.0,1.5$ simulated in a box of size $\left(L_{x}, L_{y}, L_{z}\right)=(30,5,50)$. In equilibrium, the scaling properties of polymer brushes do not depend on the way the free ends are modelled, being retained in simulations of polymer brushes in good solvent [28] or in theoretical treatments assuming all free ends are at the maximum distance from the grafting wall (Alexander model [29-31]) or can move over the whole brush thickness (self-consistent mean-field theory $[22,23])$. Regardless of the model used, and hence of the way free ends are included in the description, the interface between the brush and the rest of the channel is always smooth, as highlighted in Fig. 2 by the dashed circle. The precise configuration of the free ends thus gives no significant contribution to the brush equilibrium behavior. By contrast, it turns to be crucial if the brush is subdued to flow, since the free ends determine the brush

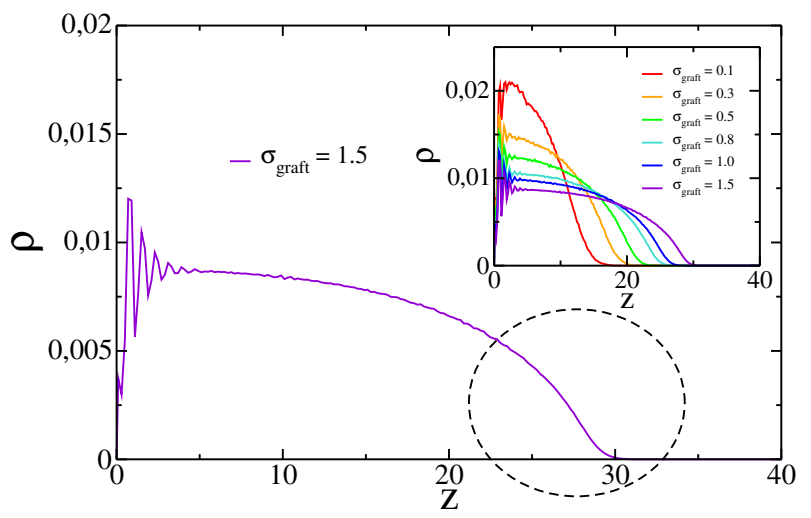

FIG. 2. Density profiles $\rho(z)$ for $n=40$. In the inset: comparison among density profiles at different grafting densities $\sigma_{\text {graft }}=0.1,0.3,0.5,0.8,1.0,1.5$. In the main frame, a zoom for $\sigma_{\text {graft }}=1.5$, with the circle highlighting the smooth decay even in the higher volume fraction case.

elastic properties in general, and the shear modulus in particular, which can couple to hydrodynamics even at low Reynolds numbers, as we will show in the following. Therefore, we stress that the smooth decay of the density profile does constitute a peculiarity of the filamentous coating and it must be considered for a correct understanding of the polymer brush under out of equilibrium dynamics.

In the following the simulation results we will present have been obtained with brushes with $\sigma_{\text {graft }}=1.5$, corresponding to a polymer brush with an average distance between filaments of $\approx 20 \mathrm{~nm}$ and a thickness in the range $300 \div 1000 \mathrm{~nm}$ to mimic the endothelial glycocalyx [20]. We also define the brush surface $S$ position as the averaged position of the farthest monomer from the grafting wall. At equilibrium, this corresponds to $S=S_{0}=29.1$ (DPD units).

\section{A. Hydrodynamic instability of the flat brush surface}

\section{Surface morphological instability has elastic origin}

It has been recently shown that under specific conditions of a parabolic flow the brush polymers co-ordinate the recursive motion of their free ends and give rise to a collective effect, namely a traveling wave over the brush surface [13]. The idea of the present study is to attribute the presence of the wave, that is, of finite-amplitude oscillations, to an instability driven by hydrodynamics.

The occurrence of surface protuberances can be attributed to a morphological instability of elastic origin. Indeed, it is quite well established that a surface subject to an axial stress (such as shear stress) stores elastic energy which can be partially released in the form of a surface deformation, which has a lower energy than a flat 
one. An example of a such a behaviour is known as the Asaro-Tiller-Grinfeld $[32,33]$ instability discussed in the context of solid surfaces [34], where the modulation takes place thanks to mass rearrangement (surface diffusion, for example). Another context where surface instabilities appear is the clamped gel problem [35], where the bottom surface of a thermoresponsive gel is fixed to a solid surface. Upon varying the temperature the gel experiences a uniaxial stress leading to surface modulation which is not due to mass rearrangement but to bucklinglike modes. Similarly, when an elastic medium is in contact with a fluid under shear flow, the elastic medium is under a uniaxial stress and a surface deformation leads to a partial release of the stress [36]. In all these three cases a linear stability analysis has been performed showing the occurrence of a surface instability. The third case is the most relevant for our study. The linear stability analysis was performed in Ref. [36]. However, no simple analytical expression is available. Here, we have simplified the analysis making it more adequate for a polymer brush instead of a gel, and precisely: (i) in Ref. [36] the gel (in our case the brush) has a visco-elastic behavior and an inertial contribution, while we neglect (legitimately) inertia and consider the brush to be purely elastic (given the fact that flow is almost absent within the brush at low $W i$ ); (ii) in Ref. [36] the gel-fluid interface is endowed with surface tension, while we show that this is not only unnecessary (a cut-off wavenumber is ensured by gel elasticity and fluid viscosity) but the notion of surface tension does not make sense; (iii) in Ref. [36] the fluid has a finite extent in z-direction, whereas here we took the assumption that the system is infinite (meaning that the system size is large as compared to wavelength of interest, which is a quite legitimate assumption in the experiments of Lanotte et al.[12] and in our numerical set-up). In both works the medium is taken to be incompressible. Our simplifying assumptions have the advantage to yield a simple analytical expression for the dispersion relation.

\section{Linear stability analysis}

Since the flow close to walls is of shear type, we consider the following set-up: an imposed linear shear flow $u_{x}=\dot{\gamma} y$ (where $\dot{\gamma}$ is the shear rate) with a geometry similar to that in Fig. 1 but with a large $L_{z}$ (actually infinite; this is legitimate as long as the brush thickness and wavelengths of interest are small as compared to $L_{z}$, two fulfilled conditions).

The fluid obeys the Stokes equations

$$
\begin{gathered}
-\nabla p+\eta \Delta \mathbf{v}=0 \\
\nabla \cdot \mathbf{v}=0,
\end{gathered}
$$

where $\mathbf{v}$ is the velocity, $p$ thee pressure and $\eta$ the viscosity. The brush is taken to be a linearly elastic domain described by the Lamé equations. Due to rotational symmetry in the plane of the planar brush-fluid interface the dispersion relation depends only on the modulus of wavenumber. For this reason it is sufficient to consider a $2 \mathrm{D}$ model (the system is supposed to be invariant in the $y$ direction). In this case it is convenient to make use of the Airy function [37] $\chi$, related to elastic stress tensor $\sigma_{i j}$ by

$$
\sigma_{x x}=\frac{\partial^{2} \chi}{\partial x^{2}}, \quad \sigma_{z z}=\frac{\partial^{2} \chi}{\partial z^{2}}, \quad \sigma_{x z}=-\frac{\partial^{2} \chi}{\partial x \partial z},
$$

The Lamé equation (equilibrium equation for an elastic medium) reduces to solving[37]

$$
\Delta^{2} \chi=0
$$

The Airy function obeys a bi-harmonic equation. Once $\chi$ is determined one can extract the stress tensor from (5), and the displacement field $\mathbf{u}$, upon using Hookes law,

$$
\begin{aligned}
& e_{x x}=\frac{1+\nu}{Y}\left[(1-\nu) \sigma_{x x}-\nu \sigma_{z z}\right], \\
& e_{z z}=\frac{1+\nu}{Y}\left[(1-\nu) \sigma_{z z}-\nu \sigma_{x x}\right] \\
& e_{x z}=\frac{1+\nu}{Y} \sigma_{x z},
\end{aligned}
$$

where $Y$ is the Young modulus, $\nu$ the Poisson ratio, and $e_{i j}=\left(\partial_{i} u_{j}+\partial_{j} u_{i}\right) / 2$ is the strain tensor.

For a planar interface the shear stress in the fluid is constant (equal to imposed one) and is equal to that in the brush (due to mechanical equilibrium). Linear stability analysis is performed by considering that the interface $z=h_{b}+h(x, t)$ is deformed with a small amplitude $\epsilon$. While the Stokes and Lamé equations are linear, the solution is a nonlinear function of $h$. Linear stability consists in retaining only linear terms in deformation amplitude. Because in a linear regime modes do not couple, it is sufficient to consider a single Fourier mode, so that interface equation reads $z=h_{b}+\epsilon e^{i q x+\omega t}+$ c.c., where $q$ is wavenumber and $\omega$ is the attenuation/growth rate of the perturbation $(\operatorname{Re}(\omega)>0$ signals an instability). c.c. stands for complex conjugate and will be omitted in what follows. All solutions of the linear problem follows the same dependence with $x$ and $t$

a. Hydrodynamic solution Taking the rotational of (3) eliminates the pressure and one obtains $\Delta\left(\partial_{z} v_{x}-\right.$ $\left.\partial_{x} v_{z}\right)=0$. Using continuity equation (4) one obtains $i q v_{x}+\partial_{z} v_{z}=0$ allowing us to express $v_{x}$ in terms of $v_{z}$. Using that $\partial_{z} v_{z}=-i q v_{x}$ (eq. (4)), implying that $\partial_{z} v_{x}=-i \partial_{z z} v_{z} / q$, one easily finds that $v_{z}$ obeys the degenerate equation

$$
\left(\partial_{z z}-q^{2}\right)^{2} v_{z}=0
$$

so that the general solution of $v_{z}$ is given by (recall the system is taken semi-infinite along $z$ and we retain only decaying solution in $z$ )

$$
v_{z}=(A+B z) e^{-q z} e^{i q x+\omega t}
$$

where $A$ and $B$ are integration constant. 
b. Elastic problem $\chi($ see $(5))$ has the following solution

$$
\chi=\left[(C+D z) e^{-q z}+\left(C^{\prime}+D^{\prime} z\right) e^{q z}\right] e^{i q x+\omega t}
$$

with integration constant $C, D, C^{\prime}$ and $D^{\prime} . v_{x}$ can be determined from the relation with $v_{z}$ evoked above, and using the Stokes equation $p$ can be determined. Once these quantities are known the hydrodynamic stress can easily be determined, using $\Pi_{i j}=-p \delta_{i j}+\eta\left(\partial_{i} v_{j}+\partial_{j} v_{i}\right)$. We have thus 6 different constant $A, B, C$ and $D$, and $C^{\prime}$ and $D^{\prime}$, plus the interface deformation amplitude $\epsilon$.

c. Boundary conditions Using the boundary conditions at the brush interface, $z=h_{b}+h(x, t)$ (and developing them to linear order in $\epsilon$ ), one obtains homogeneous algebraic equations for the amplitudes. The boundary conditions are: (i) continuity of normal stress, (ii) continuity of tangential stress, (iii) zero displacement at the bottom wal $\left(u_{x}=u_{z}=0\right)$, (iv) ) continuity of velocity $\left(v_{x}\right.$ and $\left.v_{z}\right)$ at the interface. These 6 conditions allow us to express the 6 integration constant as a function of $\epsilon$. Finally, imposing a continuity of interface velocity (given by $\left.\partial_{t} h(x, t)\right)$ with that of the fluid velocity $v_{z}$ provides an additional relation resulting in a homogeneous equation for $\epsilon$ and a nontrivial solution exists only if the prefactor is non zero (alternatively we could use that the determinant of the full system of algebraic equations be zero for a non trivial solution to exist). This results in a dispersion relation relating $\omega, q$ and control parameters. In the limit of a Poisson ratio $\nu=1 / 2$ (incompressible gel) the dispersion relation takes a relatively simple form (after algebraic manipulations which are straightforward)

$$
\begin{aligned}
& {\left[q^{2}+1-\cosh ^{2}(q)\right] \omega^{2}-2 \omega \sinh (q) \cosh (q)} \\
& +i \frac{W i q^{2}}{3}-q^{2}-\cosh ^{2}(q)=0
\end{aligned}
$$

where $q$ is the dimensionless wavenumber related to the physical wavenumber $\tilde{q}$ by $q=\tilde{q} h_{b}$ (we recall that $h_{b}$ is the brush thickness) and $W i$ will be referred to as the Weissenberg number defined by $W i=\dot{\gamma} \eta / G$. In this definition for $W i$ we have equated $t_{\text {flow }}=(\dot{\gamma})^{-1}$ and $t_{\text {brush }}=\eta / G$. We have also used the relation between the Young modulus $Y$ and shear modulus $G, Y=3 G$ (for incompressible material). The physical growth rate $\tilde{\omega}$ has been scaled by the shear rate, so that in Eq. (11) the growth rate $\omega$ is dimensionless and reads $\omega=\tilde{\omega} / \dot{\gamma}$. It has a real and imaginary part and it will be written as $\omega=\omega_{r}+i \omega_{i}$. An instability is signaled by a positive value of $\omega_{r}$. A typical shape of $\omega_{r}(q)$ is given in Fig. 3(a). We can notice that there is a critical value of $W i$ beyond which there is a band of wavenumbers corresponding to positive $\omega_{r}$, signaling an instability of the surface. We see that the critical value of $W i$ is around 25 , meaning that for an instability to arise in our simple model the shear stress should be about 25 times larger than the bulk shear modulus $G$. Note that we are considering an incompressible brush, so that at $q=0, \omega_{r}=-\infty$, meaning that any homogeneous $(q=0)$ compression of the brush is stable, its relaxation time being instantaneous. In reality a brush has a finite compressibility, so that at $q=0$ we expect $\omega_{r}$ to take a finite value. This means that in reality the curves in Fig. 3(a) will be shifted upwards and hence the critical value of $W i$ will be smaller. In other words, we predict that the instability of a real (compressible) brush would take place when the shear stress reaches values of few times the elastic modulus.

The above linear study has an indicative value showing the existence of an instability and of the accompanying band of active modes (unstable modes). While it may be possible to extend the model to explicitly take into account the compressibility of the brush, the elastic response of the brush considered here, which will be discussed in the next section, has a nonlinear response in a quite large range of shear stress. Therefore a full quantitative theory would require the development of a nonlinear elastic theory for the brush to take on the study of its linear and nonlinear instability with respect to surface modulation, which is beyond the goal of the present paper.

We note in passing that the smallest wavelength below which the surface is stable $\left(\lambda_{2}\right.$, corresponding to the largest value of $q$ at which the upper curve in Fig. 3(a) crosses the $q$-axis), represents a "microscopic" cut-off suppressing short wavelength instabilities. Usually the presence of a cut-off is connected to surface tension [36]. By contrast, here the cut-off length is controlled by the brush elastic modulus. The presence of a cut-off means that, if the simulation box $L_{x}$ is smaller than $\lambda_{2}$, then no instability mode can fit in the box and the surface is stable. We will see numerically in section III B that increasing the box size $L_{x}$ the surface becomes morphologically unstable and that this instability causes a strong flow reduction, as observed experimentally [12].

\section{Linear and non linear elastic response of the brush}

In the model sketched above we have treated the brush as a linear incompressible elastic medium (Poisson ratio is set to $1 / 2$ ). We have attempted to investigate this matter further by analyzing numerically the response of the brush to an imposed stress. Figure 4(a) shows the relationship between an imposed compression and the corresponding stress response of the brush. We simulate a Surface Force Apparatus experiment and compress the brush by mean of an ideal plate posed at a certain distance from the grafting wall; the plate behaves as a rigid wall of infinite mass for monomers, while it is transparent to solvent particles. The stress is estimated as $P=F / A$, where $F$ is the time average of the total DPD conservative force exerted by each monomer on the plate surface of area $A$. In Fig.4(b), on the other hand, we show the relation between a shear stress exerted on the brush and the corresponding shear strain. The shear stress $\sigma$ is imposed by the flow and it is calculated as $\sigma=\eta \dot{\gamma}$, where $\dot{\gamma}=v_{\max } / z_{\max }$ with $v_{\max }$ the maximum velocity mea- 


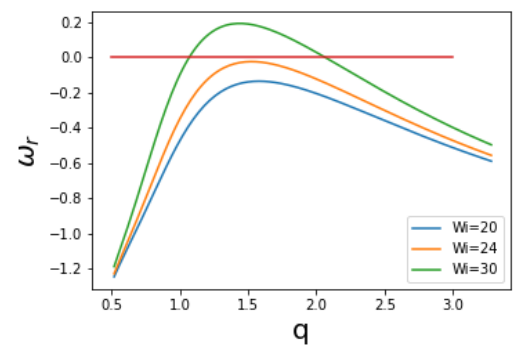

(a)

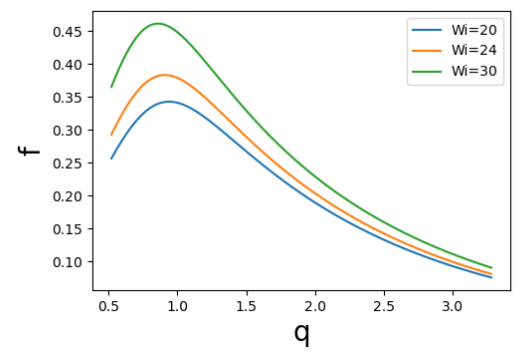

(b)

FIG. 3. Real and imaginary parts of the growth rate $\omega$ as a function of wavenumber $q$ for different values of $W i$ : a) the dispersion relation showing real part of $\omega ; \mathrm{b}$ ) the frequency $f$ obtained from the imaginary part of $\omega$ divided by $2 p i$.

sured at the corresponding $z=z_{\max }$. We recall that for our system $\eta=0.84$ in DPD units. The shear strain is the average of the component along the flow direction of the end-to-end vector, $\Delta x=\left\langle R_{e e, x}\right\rangle$. It is evident from the two figures that those relations are linear for small strains only. As a consequence, the polymer brush has a nonlinear elastic character that comes into play as the imposed flow gets higher, and it gets stiffer and stiffer on increasing the rate of compression/strain.

In the region of small deformations, we extract the values of the two elastic moduli, $Y$ and $G$. A linear fit yields $Y=1.4$ and $G=0.59$ and hence $\nu \approx 0.19$, confirming the compressibility of the brush.

We also estimate the shear stress at the brush outer surface layer $S$ as $\sigma_{S}=\eta d v(z) /\left.d z\right|_{z=S}$, where $d v(z) /\left.d z\right|_{z=S}$ is obtained from the linear slope of the velocity profile $v(z)$.

Here we follow Ref. [13] wher it has been shown that the wave appears when the velocity profile exhibits a flow inversion close to the brush surface. Figure 5 shows the minimum of the velocity profile as a function of $W i$ for the system with $\left(L_{x}, L_{y}, L_{z}\right)=(30,5,50)$. The Weissenberg number in our simulation is calculated as $W i=\eta / G \dot{\bar{\gamma}}$, where $\overline{\dot{\gamma}}=v_{\max } /\left(z_{\max }-h_{b}\right)$ is a measure of the imposed flow. Indeed, even if the imposed flow is parabolic, in the brush vicinity the fluid velocity is close to zero and the flow can be legitimately linearized. The data show that the flow inversion, and hence the appearance of the surface wave, happens at $W i \approx 0.25$, which corresponds to $\sigma_{S} \approx 0.3$ (see inset). This value is comparable with the value of $G$ reported above. In other words, the instability takes place as soon as the brush experiences at its surface a shear stress comparable with its shear modulus $G$. As we have seen in the dispersion relation (11), $W i$ is the only parameter entering there, and we have seen that the instability takes place for $W i \sim 1$ (meaning that the shear stress is of the order of the shear modulus). Thus, we indicate in the following relation

$$
\sigma_{S} \sim G
$$

the necessary condition for the onset of such instability. Below such threshold we expect the polymer brush to reduce the flow as if it was a regular rigid wall, without inducing any anomaly in the velocity profile.

The dimensionless fastest growing wave number has a value around 1.4 (see Fig.3(a)) corresponding to a wavelength $\lambda=2 \pi h_{b} / 1.4 \sim 110$ (in DPD units). Referring to figure 6 , showing the first coefficients of the Fourier power spectra in the spatial domain, we find that for the $L_{x}=180 \mathrm{~s}$, the dominant harmonic corresponds to a wavelength which is half the system length (a value

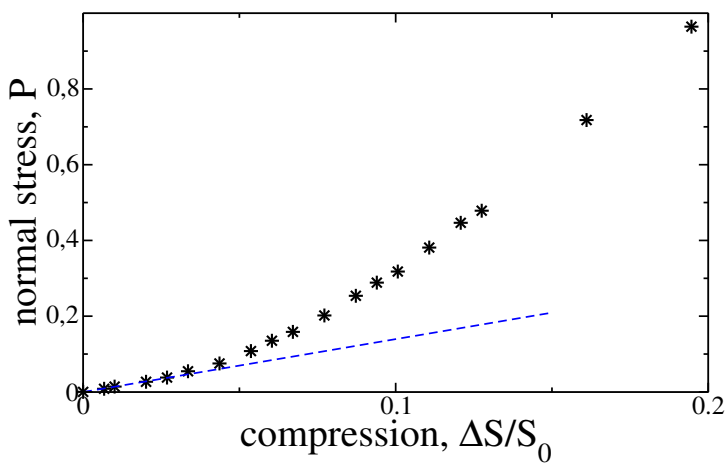

(a)

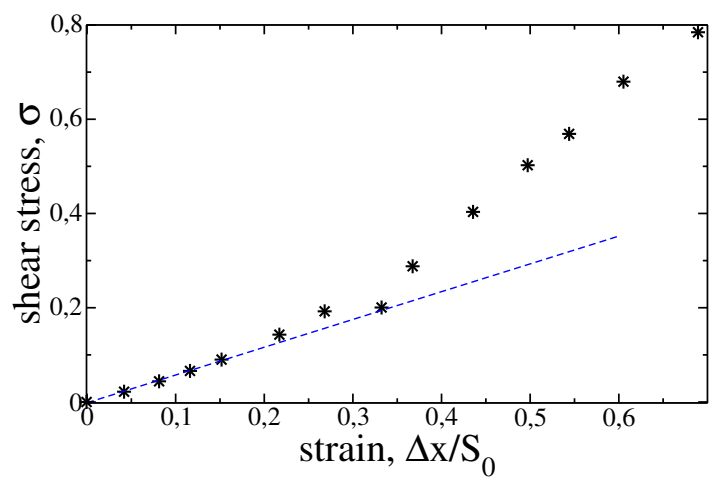

(b)

FIG. 4. The brush nonlinear response to compression (a) and to strain (b). $S_{0}$ is the averaged position of the farthest monomer from the grafting wall at equilibrium. As the applied normal or tangential load increases, the brush stiffens. The blue lines are linear fits in the region of small deformations yielding an estimation of the brush Young modulus (a) and shear modulus (b). 


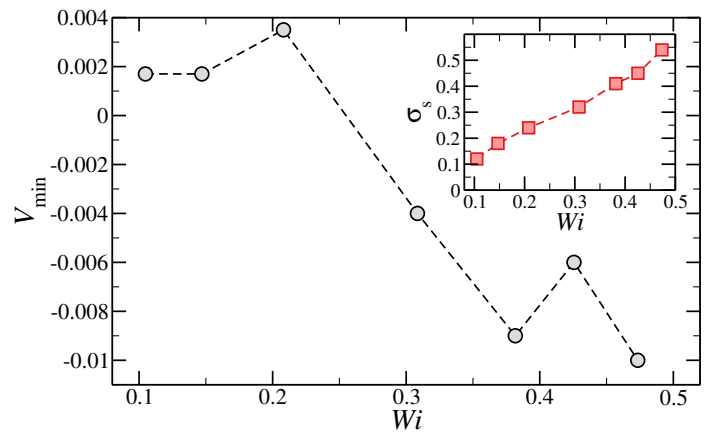

FIG. 5. The minimum of the velocity profile as a function of the Weissenberg number $W i$ for the $\left(L_{x}, L_{y}, L_{z}\right)=(30,5,50)$ system. The inset shows the dependence of the shear stress at the brush outer surface layer, $\sigma_{S}$, as a function of $W i$.

of 90), thus comparable to the theoretical value 110 . While in the $L_{x}=30$ the dominant wavelength is basically selected by the system size. Indeed, instead of fastest mode, the smallest wavelength (and corresponding to an unstable mode) fitting in the simulation box can be exhibited. In the vicinity of instability threshold, this wavenumber remains comparable to that corresponding to the fastest one (see Fig.3(a)), and thus larger than the box size, explaining the absence of instability for $L_{x}=30$.

Furthermore, as explained in Ref. [13], the instability manifests itself as a travelling wave. The linear stability presented here shows that $\omega$ has an imaginary part (see Fig.3(b)), and thus the modulation has a travelling wave character. The frequencies extracted from the linear analysis are reported in Fig.3(b) and are in good agreement with the ones obtained from simulations (see Fig.4.(c) of Ref. [13]). To compare those results we should first notice that the Weissenberg number used in Ref. [13] is different from the $W i$ defined in this paper and that the range $\mathrm{i}\{137,218\}$ in Ref. [13]) corresponds to our $W i=\{0.31,0.47\}$. Then, it is sufficient to scale the frequencies shown in Ref. [13] by the factor $\eta /(G * W i)$ to obtain frequencies dimensionalized by shear rate in the order of 0.2 (in DPD units), therefore in the same order of magnitude as those appearing in Fig.3(b). In addition, since the wavelengths in simulation and theory are comparable, we conclude that the travelling speeds obtained numerically and analytically agree with each other in terms of order of magnitude.

The prominence of the shear moduli $G$ compared to other elastic moduli in determining brush instabilities resides in the observation that a polymer brush, and polymers in general, can be more easily sheared than compressed. In our simulations we find $Y / G \approx 2$, but it is worth noticing that in real polymer brushes $Y / G \approx O\left(10^{2}\right)[38,39]$. As a result, a hydrodynamic load more easily excites shear modes than compression modes. The gap between those two elastic features is not surprising if we consider how anisotropic a polymer brush

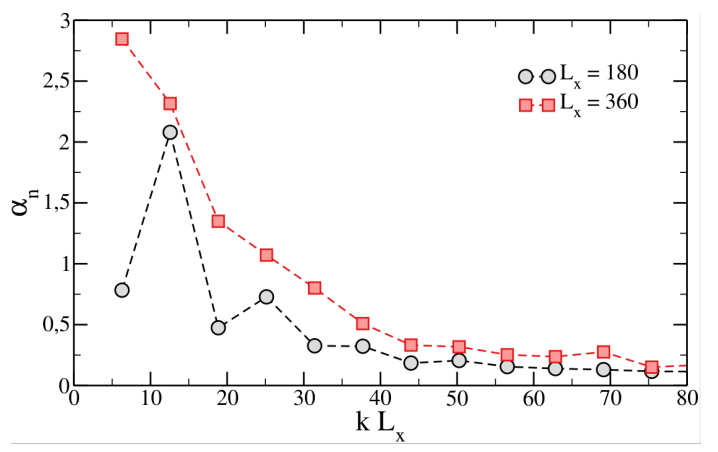

FIG. 6. Spatial Fourier coefficients of surface modulation for different system sizes.

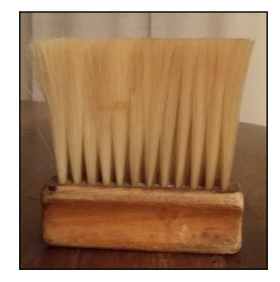

(a)

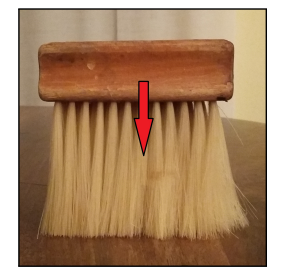

(b)

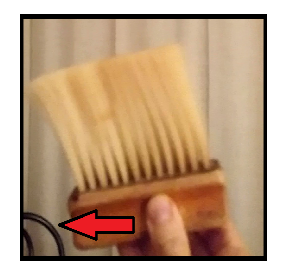

(c)

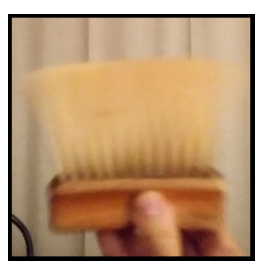

(d)

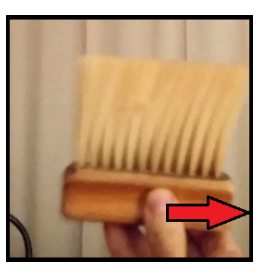

(e)
FIG. 7. Elastic properties of a macroscopic brush, which serve as guidelines to infer polymer brush elastic properties: (a) filaments can sustain the weight of the wooden substrate, meaning that the Young modulus is not negligible; (b) the brush is easily shearable, as shown by the deformed filaments, together oscillating under a light shear impressed by a lateral movement.

is, by construction. Figure 7 shows a series of images of a macroscopic brush (Fig. 7(a)) that highlights this property: although the brush can sustain the weight of the wooden substrate (Fig. 7(b)), revealing a non-negligible compression resistance, it can be easily sheared just by slightly left-right shaking one hand (in Fig.7(c)-7(e)).

\section{B. Flow reduction in the bulk channel as effect of the brush surface instability}

An interesting experimental result was reported regarding the influence on flow properties of a polymer brush coating the internal surface of a glass capillary[12]. Given a pressure-driven flow, one would expect the maximum velocity in the capillary center to be that given by a Poiseuille profile with a radius reduced by the brush 
thickness. By contrast, the measured maximum velocity was found to be less than expected, as if the brush were twice thicker than its real value. In terms of numerical values, let us denote by $v_{0}$ the maximum velocity for the bare capillary (in the absence of the brush), and $v_{0}^{\prime}$ that of a capillary having a radius equal to the bare value minus the brush thickness (as if the brush effect was to only reduce the capillary radius). The measured velocity [12] $v_{0}^{\prime \prime}$ was found to be lower than $v_{0}^{\prime}$ by $30 \%$. Our numerical results show that that the morphological instability, discussed above, is a plausible candidate to explain this behavior.

We find that wherever a surface wave is present, a significant reduction of the bulk maximum velocity as function of the channel length is also observed. We first recall that in a channel the expected fluid velocity profiles have a parabolic shape, as analytically obtained in case of Stokes flow in a slit-pore geometry. Since in our simulations polymers have been grafted only at the $z=0$ wall, the centre of the parabola does not coincide with the centre of the channel. Parabolas are thus fitted by

$$
v_{x}(z)=\frac{\rho A}{2 \eta}\left(\left(R_{\max }-\mathcal{A}\right)^{2}-(z-\mathcal{A})^{2}\right),
$$

where the pressure gradient has been substituted by the imposed force per unit volume $\Delta P / L=\rho A, R_{\max }$ is the $z$-coordinate corresponding to the maximum velocity and $\mathcal{A}$ is a fitting parameter. If there is no wave at the brush surface we find $\mathcal{A} \sim h_{b}$ and the velocity profile (Fig. 8, black curve) can be almost perfectly overlapped with the velocity profile of a channel of width $L_{z}-h_{b}$ (Fig. 8, orange curve). This means that if no instability is present, the polymer brush behaves as a rigid compartment which effectively reduces the channel width. We see in Fig. 3(a) that for $W i \sim 30$ the largest values of $q$ beyond which there is no instability is of about 2 (changing $W i$ does not affect to much this value, and we have checked from our dispersion relation that for $W i=100$ the threshold is of about 3). This provides a cut-off length, which is the minimum box length below which no instability should be present, of about $L_{\min }=2 \pi h_{b} / 2=\pi h_{b} \sim 78$. We find that, while for $L_{x}=30$ there is no instability, increasing the channel length from $L_{x}=30$ to $L_{x}^{\prime}=6 L_{x}=180$ results in a pronounced decrease of the fluid bulk maximum velocity, which is associated to a surface instability. Under these conditions the maximum velocity is around $60 \%$ lower than that obtained when no instability is present and the brush is flat $\left(L_{x}=30\right)$. Further increasing the box size can only increase this figure, since more wavelengths can be excited as the channel's length grows. Coherently with this picture, Fig. 8 shows that $v_{\max }$ slightly decreases when the length of the channel doubles $\left(L_{x}^{\prime \prime}=12 L_{x}=360\right)$.

We propose that the hydrodynamics-brush coupling modifies the energy balance: the flow excites one or more natural mode frequencies of the brush and the supplied energy, instead of increasing the bulk fluid velocity, feeds the instability. Therefore, the brush motion is enhanced,

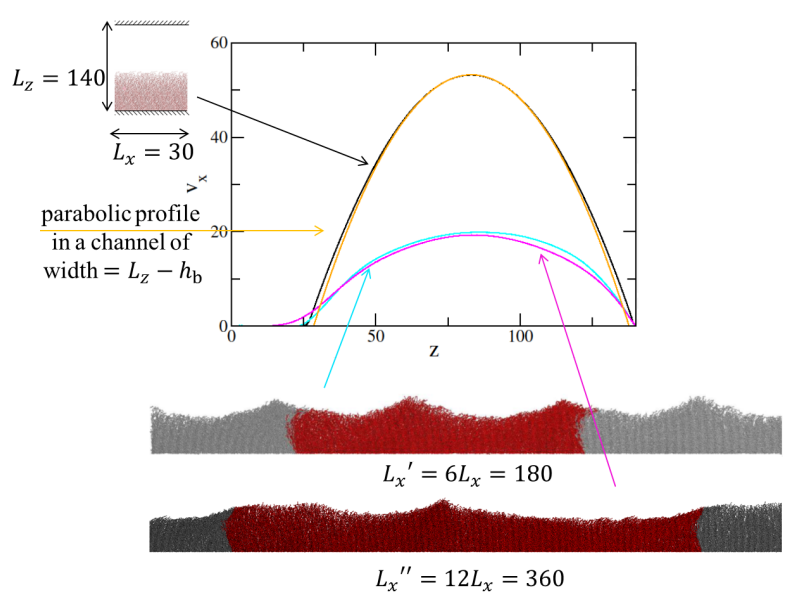

FIG. 8. Comparison between the flow profiles inside channels of increasing lengths: $L_{x}=30$ (black curve), $L_{x}=180$ (turquoise curve) and $L_{x}=360$ (violet curve). The width is fixed at $L_{z}=140$ and the imposed acceleration is $A=0.01$ in all cases. The orange plot corresponds to the parabolic velocity profile in a channel of reduced width $L_{z}-h_{b}$. It is observed a significant reduction of the maximum velocity as function of the channel length. The trend shows a saturation around a $60 \%$ decrease, associated with the surface wave. We show pictures of the brush surface wave which settles in the longer systems (in red color the simulated system, with its nearest replicas in grey, to convey the impression of the periodicity of the surface modulation). $W i=1.29$ in all cases.

as revealed by the aspect of the wave (see Fig. 8). This viewpoint is in line with the outcome of an experiment by Kumaran and Muralikrishnan devoted to the characterization of viscous flow past a soft interface: beyond a certain threshold for the applied shear stress, the transport of energy disfavours the average flow and an increase in the fluid apparent viscosity is measured [40], which is coherent with the decrease of the maximum velocity we show in Fig. 8. Again, this analogy is an additional hint that polymer brushes can develop hydrodynamic-induced instabilities. Interestingly, thanks to the mesoscale nature of our simulations we are able to directly observe the oscillations of the soft interface, which were only inferred in Ref. [40].

\section{Comparison with experiments}

We have seen above that that the morphological interface instability can drastically reduce the flow, which in our simulations is decreased by $\approx 60 \%$ compared to the planar case. However, Lanotte et al. [12] measured only $\mathrm{a} \approx 30 \%$ reduction. A possible reason for this discrepancy is the polydisperse nature of the brush investigated in Ref. [12]. On a qualitative level it has been shown that polydispersity changes how the surface wave appears and 
behaves [20], although a thorough quantitative investigation of its effects is beyond the scope of the current work.

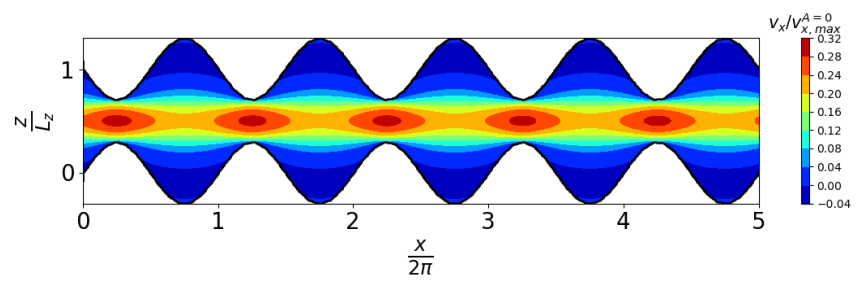

(a)

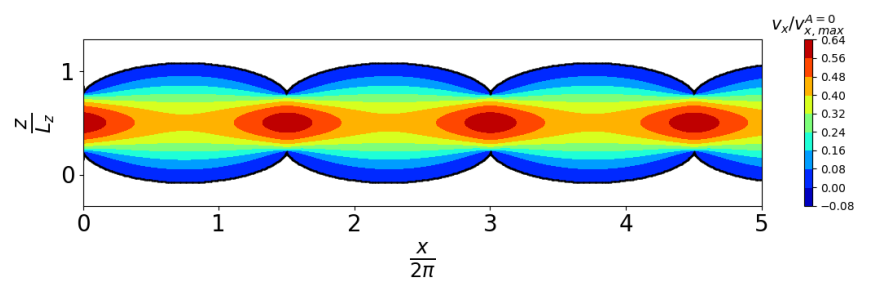

(b)

FIG. 9. Velocity pattern in the channel with a modulation of interfaces: (a) a sinusoidal modulation, with $z_{\mathrm{top}} / L_{z}=$ $A \sin (-x /(2 \pi))+1$ for the upper wall and $z_{\text {bottom }} / L_{z}=$ $A \sin ((x / 2 \pi))$ for the bottom wall, and (b) a cycloidal modulation, with $z_{\mathrm{top}} / L_{z}=A / 2(1-\cos (-x /(2 \pi))+1$ for the upper wall and $z_{\mathrm{bottom}} / L_{z}=-A / 2(1-\cos ((x / 2 \pi))$ for the bottom wall. The color code refers to the modulus of the velocity. Here $A=0.3$ in unit of the channel width.

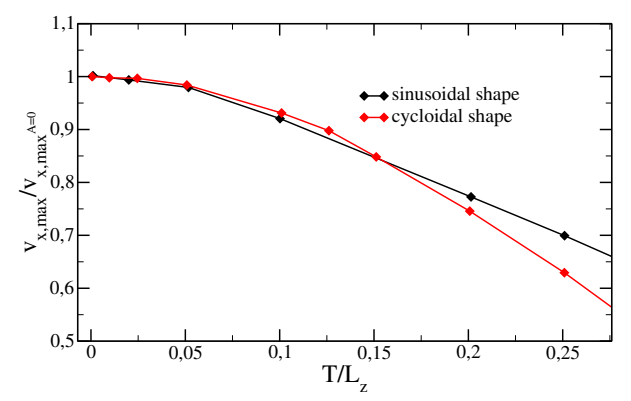

FIG. 10. Maximum velocity divided by that corresponding to a straight channel as a function of surface deformation. Comparison between the sinusoidal wall modulation (black) and the cycloidal one (red). Notice that the modulation thickness $T$ reported on the $x$-axis is $T=A$ for the cycloidal case and $T=2 A$ for the sinusoidal case.

Another likely source of difference between the numerical and experimental results is provided by system size and interface geometry. Because of computational costs our system size along the flow direction cannot be as long as it is in real experiments. Indeed, with the level of description we chose the lateral size $L_{x}$ cannot be more than a few (2 to 4$)$ times the instability wavelength. This means that higher wavelength modes can not fit in the simulation box, and this will most likely affect the nonlinear evolution of the surface wave, such as its amplitude, and its temporal dynamics. Moreover, as we have previously observed in Fig. 8, the more the velocity reduction the more the deviation of the wave front from a simple sinusoidal shape. Here, we have attempted to extract the role of interface geometry for the maximum velocity change. We used a Lattice Boltzmann simulation [41] to draw the flow field between two rigid walls which display a fixed modulation mimicking the brush deformation. We have focused on two cases, as shown in Fig. 9: a sinusoidal modulation and a cycloidal modulation. The main results are reported in Figure 10, which shows the evolution of the maximum velocity as a function of the deformation amplitude $A$ and compares the two geometries. We see that a velocity reduction of $\approx 25 \%$ (closer to experiments) is obtained for a wall modulation which is $20 \%$ of the channel width. However, in the cited experiments [12] such velocity reduction is found for a brush thickness which is only $5 \%$ the channel width. This indicates that it is likely that the anomaly in the maximum velocity measured actually comes from an interplay between the brush and the flow and it is not only a matter of geometry of the boundary. This bolster the idea of the elastic instability as the leading mechanism destroying more the flow field with respect to what expected for the flow field past rigid bodies at low Reynolds numbers.

\section{CONCLUSION}

We have discussed the behavior of a polymer brush as exposed to a parabolic flow in slit pore geometry. Our mesoscale investigation accounts for the specific dynamics of the polymer free-ends exposed to flow: driven by the long-range of hydrodynamics, they can generate collective effects, namely a travelling wave at the brush surface. By analogy with solid elastic films we have quantified the Young and shear moduli and indicated the wave as the onset of a shear instability induced by hydrodynamics. We have found a criterion for the onset of the instability in the following condition:

$$
\sigma_{S} \sim G
$$

where $\sigma_{S}$ is the shear stress exerted by the flow on the brush surface $S$ and $G$ is the brush shear modulus. Such an outcome is in line with a previous theoretical result [36] found for flow past a soft gel and widen the range of hydrodynamic interactive soft media to embrace polymer brushes too. We stress on the importance of $G$ in determining the threshold: this effect can be traced back to the difference between the values of the two moduli, with the shear modulus being much smaller than $Y$, differently from what is expected in standard solid matter for isotropic materials. Beyond such threshold, brush instabilities can take place and waves can form. Moreover, in the presence of waves of big oscillation amplitudes, the imposed pressure drop feeds the instability, resulting in a strong flow reduction in the channel. We thus offer a numerical counterpart to the microfluidic experiments of 
Ref.[12], providing a long-sought plausible interpretation of the results reported therein. We emphasize such interpretation, since we have also proved that a rigid interface model is not a good approximation for a polymer brush under flow: in the end, at low Reynold numbers, a polymer brush, with its dynamical protrusion inside the fluid, displays a richer dynamics than a sharply-cut interface does, even tailoring its geometry for much complicated shapes.

It is worthwhile noticing that our case study can be related to blood circulation: the chosen set-up resembles the microcapillary environment and the simulated polymer brush is a simpler model for the endothelial glycocalyx. Interestingly, the shear modulus of the endothelial glycocalyx layer has been measured to be $G=6.7 \mathrm{~Pa}$ [39] and at physiological conditions shear stresses at the microcapillary walls are of the same order of magnitude (few Pascals). Thus, the criterion condition of Eq.(12) can be fulfilled and the surface wave we have discovered might come into play under physiological conditions. We speculate that if the discussed instability and flow reduction do take place at the microcirculation level, they could serve as a control in regulating the blood velocity and hence the shear stress felt by the vascular walls.

As outlined in Figs. 4, a polymer brush in not simply linearly elastic: it becomes stiffer and stiffer on increasing the $W i$. Moreover, the properties of the brush also depends on temperature (or, equivalently, solvent quality) [42]. Thus, a full clarification of the brush phenomenology will require a more complex model encompassing nonlinear dynamic sources and thermal effects. We remark that our DPD simulations contain almost one million particles and that a further increase of the system size is frustrated by computational limits. The construction of a continuum model to solve the NavierStokes equations with adequate boundary condition to mimic the brush layer in order to investigate the behavior also in case of flowing objects is an interesting task for future investigations.

\section{ACKNOWLEDGEMENTS}

We thank CNES (Centre National d'Etudes Spatiales) (C.M. and L.B.) for a financial support and for having access to experimental data, and the French-German university program "Living Fluids" (grant CFDA-Q1-14) (C.M.)

\section{Appendix: details on the DPD method}

DPD is a coarse-graining of MD, employing both spatio-temporal averaging of interaction potentials as well as grouping of atoms into single particles (the DPD particle). It was introduced by Hoogerbrugge and Koelman in 1992 [14] to simulate isothermal Navier-Stokes equations. It thus grasps hydrodynamics interactions and covers bigger timescales and bigger lengthscales than the usual ones reproducible with MD.

Each of the $N$ point-like DPD particles evolves in time according to Newton's equation

$$
m \dot{\vec{v}}_{i}=\sum_{j \neq i}^{N} \vec{F}_{i j}
$$

(with obvious choice for symbols). The forces $\vec{F}_{i j}$ acting on pairs have three contributions: a conservative one, $\vec{F}^{C}\left(r_{i j}\right)$, a dissipative one, $\vec{F}^{D}\left(r_{i j}\right)$, and a stochastic one, $\vec{F}^{S}\left(r_{i j}\right)$. These are all central forces such that $\vec{F}_{i j}=-\vec{F}_{j i}$, thereby guaranteeing local momentum conservation. All of them act within the same distance range $r_{c}$, that is chosen as the lengthscale unit. The conservative force has the following expression:

$$
\vec{F}^{C}\left(r_{i j}\right)= \begin{cases}a_{\alpha \beta}\left(1-\frac{r_{i j}}{r_{c}}\right) \hat{r}_{i j} & r_{i j} \leq r_{c} \\ 0 & r_{i j}>r_{c}\end{cases}
$$

with the definitions $\vec{r}_{i j}=\vec{r}_{i}-\vec{r}_{j}$ for the vector distance between the $i$-th and $j$-th particle, $r_{i j}=\left|\vec{r}_{i j}\right|$ and $\hat{r}_{i j}=\vec{r}_{i j} / r_{i j}$. It is a conservative soft-core repulsive force, corresponding to the following potential

$$
V\left(r_{i j}\right)= \begin{cases}-a_{\alpha \beta}\left(r_{i j}-\frac{r_{i j}^{2}}{2 r_{c}}-\frac{r_{c}}{2}\right) & r_{i j} \leq r_{c} \\ 0 & r_{i j}>r_{c}\end{cases}
$$

where the constant value $a_{\alpha \beta} r_{c} / 2$ has been added to shift the potential to zero at $r=r_{c}$. According to standard DPD, the functional shape of Eq. (A.2) comes from averaging a Lennard-Jones 12-6 potential over short time scales and small lengthscales, thus measuring the effective potential between clusters of liquid molecules [16]. Fast dynamics (the number of collisions with neighbors, known as the cage effect of simple liquids) is averaged out, resulting in a purely-repulsive interaction. The softness is justified by the blob nature of DPD particles that, containing also empty space, should have the possibility to overlap. We note that the absence of divergences in Eq.(A.3) entails one of the advantages of DPD with respect to MD: while an hard-core potential imposes a maximum integration time-step to avoid excessive forces, the soft-core interaction allows for bigger time step values. The constant $a_{\alpha \beta}$ measures the force between two completely overlapping particles, where the indexes $\alpha, \beta$ indicate the particle type (solvent or polymer, in our case). It controls the density fluctuations of the system and it is casted from the compressibility of the modeled fluid [43].

The two other extra forces account for the loss of details in the coarse-graining procedure, namely the huge amount of collisions occurring between real molecules and constituting the microscopic foundation of transport properties, such as viscosity and diffusivity. The dissipative force takes the following form:

$$
\vec{F}^{D}\left(r_{i j}\right)=-\gamma w^{D}\left(r_{i j}\right)\left(\hat{r}_{i j} \cdot \vec{v}_{i j}\right) \hat{r}_{i j},
$$


where the "weight function" $w^{D}\left(r_{i j}\right)$ we used is:

$$
w^{D}\left(r_{i j}\right)= \begin{cases}\left(1-\frac{r_{i j}}{r_{c}}\right)^{2} \hat{r}_{i j} & r_{i j} \leq r_{c} \\ 0 & r_{i j}>r_{c} .\end{cases}
$$

Precisely, Eq.(A.4) accounts for the energy loss, introducing a friction among particles proportional to the relative velocity $\vec{v}_{i j}=\vec{v}_{i}-\vec{v}_{j}$ and to a factor $\gamma$ that depends on the temperature $T$. To balance the dissipative force, a random force is also necessary:

$$
\vec{F}^{S}\left(r_{i j}\right)=\sigma w^{S}\left(r_{i j}\right) \theta_{i j}(\Delta t)^{-\frac{1}{2}} \hat{r}_{i j}
$$

where $\sigma$ is related to temperature, $w^{S}\left(r_{i j}\right)$ is another weight function and $\theta_{i j}$ is a random number extracted from a gaussian distribution that has zero average $\left\langle\theta_{i j}(t)\right\rangle=0$ and is uncorrelated in time and among particle pairs: $\left\langle\theta_{i j}(t) \theta_{l m}\left(t^{\prime}\right)\right\rangle=\left(\delta_{i l} \delta_{j m}+\delta_{i m} \delta_{j l}\right) \delta\left(t-t^{\prime}\right)$. The condition $\theta_{i j}=\theta_{j i}$ has to be verified in order to locally conserve momentum. The factor $1 / \sqrt{\Delta t}$ in Eq. (A.6) comes from the discretization procedure of a Wiener process and ensures that the particle self-diffusion coefficient is independent from the time step value [43]. The two forces $\vec{F}^{D}\left(r_{i j}\right)$ and $\vec{F}^{S}\left(r_{i j}\right)$ act as a thermostat.

It has been proved that to reproduce the correct equilibrium probability distribution of the NVT ensemble the dissipative and random forces have to verify two constraints [44]:

$$
w^{D}\left(r_{i j}\right)=\left[w^{S}\left(r_{i j}\right)\right]^{2} \quad \text { and } \quad \gamma=\frac{\sigma^{2}}{2 k_{B} T} .
$$

In addition to these basic DPD forces, other kinds of interaction can be included in the DPD frame according to the specific simulated system. In the present case, to mimic polymers, linear chains of DPD particles are connected by a finitely extensible nonlinear elastic potential (FENE) [18]

$$
\vec{F}_{i j}^{F E N E}=-2 k R^{2} \frac{r_{i j}-r_{e q}}{R^{2}-\left(r_{i j}-r_{e q}\right)^{2}} \hat{r}_{i j} \quad r_{i j}-r_{e q}<R
$$

where $k$ is the spring constant, $r_{e q}$ the neighbor equilibrium distance and $R$ the maximum allowed extension. An extra monomer is added to each chain as anchor, i.e. grafting point. Grafting point coordinates are randomly chosen from a uniform distribution and located on a flat surface $z=z_{\text {wall }}$ according to the imposed grafting density $\sigma_{\text {graft }}$, defined as $\sigma_{\text {graft }}=N_{c h} /\left(L_{x} L_{y}\right)$ with $N_{c h}$ the number of chains composing the brush. The anchor centers of mass are aligned with the wall $z$ coordinate and their position is not updated. Note that polymers are non-ideal ones since they also interact via the DPD repulsive force, that operates as an excluded volume potential.

Given the softness of the conservative interaction potential, a repulsive force of the kind in Eq. (A.2) is not enough to prevent particles from escaping the box. It is then necessary to implement an additional mechanism to bring back particles that cross the wall line and we opted for the bounce back reflection, in which the whole particle velocity vector is reversed $\vec{v} \rightarrow-\vec{v}$. The bounceback mechanism, by construction, is consistent with the no-slip condition at the wall.

We integrate the equations of motion with a modified Velocity Verlet algorithm, as commonly done in DPD simulations [43].

We chose the DPD units such that $r_{c}=1, m_{i}=1$ and $k_{B} T=1$. The integration time step is $\Delta t=0.02$. We underline that such value is at least one order of magnitude bigger that the usual ones set for MD simulations and, thanks to the softness of the potential, is enough to make the system reach the equilibrium/steady state. We set the system number density $\rho=N /\left(L_{x} L_{y} L_{z}\right)=$ 3 [43]. The number of monomers per chain is set to $n=40$ and the grafting density $\sigma_{\text {graft }}$ spans from $\sigma_{\text {graft }}=0.1$ to $\sigma_{\text {graft }}=1.5$. Since the total number of DPD monomers $N_{m}$ is subtracted to the total amount of particle $N$, the actual number of solvent particles is $N_{s}=N-N_{m}=\rho L_{x} L_{y} L_{z}-n \sigma_{\text {graft }} L_{x} L_{y}$. Following Ref. [43], we fix the solvent-solvent interaction parameter $a_{S S}$ relating it to the dimensionless compressibility of water $\kappa_{T}^{-1}=1 /\left(\rho k_{B} T \kappa_{T}\right)$, where $\kappa_{T}$ is the fluid compressibility, then $a_{S S}=75 k_{B} T / \rho r_{c}^{4}=25$. We assume that the polymer-polymer interaction parameter has the same value (e.g. $\left.a_{S S}=a_{P P}\right)$, while we select a smaller value for the solvent-polymer parameter $a_{S P}=20(\mathrm{good}$ solvent conditions). For the interaction with walls we set $a_{w}=6<a_{S S}$ to avoid an artificial depletion zone along the walls. The noise amplitude is fixed to $\sigma=3$, as in previous literature [43]. It is worth noticing that with higher values for $\sigma$ the code takes more time to reach the equilibrium/steady state. For the FENE potential we use $r_{e q}=0.86, R=1$ and $k=50$.
[1] P. Pincus, Macromolecules 24, 2912 (1991).

[2] F. Lo Verso, S. A. Egorov, A. Milchev, and K. Binder, The Journal of Chemical Physics 133, 184901 (2010).

[3] M. Chen, W. Briscoe, S. Armes, and J. Klein, Science 323, 1698 (2009).

[4] S. N. Ramakrishna, R. M. Espinosa-Marzal, V. V. Naik, P. C. Nalam, and N. D. Spencer, Langmuir 29, 15251
(2013).

[5] B. M. van den Berg, H. Vink, and J. A. Spaan, Circulation Research 92, 592 (2003).

[6] O. Nilsson and K. G. Nygren, Upsala Journal of Medical Sciences 77, 3 (1972).

[7] R. Nakamura, T. Katsuno, Y. Kishimoto, S. Kaba, M. Yoshimatsu, M. Kitamura, A. Suehiro, N. Hiwatashi, 
M. Yamashita, I. Tateya, and K. Omori, Scientific Reports 10 (2020).

[8] E. Damiano, B. Duling, K. Ley, and T. Skalak, Journal of Fluid Mechanics 314, 163 (1996).

[9] S. Weinbaum, X. Zhang, Y. Han, H. Vink, and S. C. Cowin, Proceedings of the National Academy of Sciences 100, 7988 (2003).

[10] M. Müller and C. Pastorino, EPL (Europhysics Letters) 81, 28002 (2008).

[11] F. Léonforte, J. Servantie, C. Pastorino, and M. Müller, Journal of Physics Condensed Matter 23, 184105 (2011).

[12] L. Lanotte, S. Guido, C. Misbah, P. Peyla, and L. Bureau, Langmuir 28, 13758 (2012).

[13] S. Biagi, L. Rovigatti, F. Sciortino, and C. Misbah, Scientific Reports 6, 22257 (2016).

[14] P. Hoogerbrugge and J. M. V. A. Koelman, Europhysics Letters 19, 155 (1992).

[15] P. Español, Phys. Rev. E 52, 1734 (1995).

[16] B. M. Forrest and U. W. Suter, The Journal of Chemical Physics 102, 7256 (1995).

[17] R. M. Füchslin, H. Fellermann, A. Eriksson, and H.J. Ziock, The Journal of chemical physics 130, 214102 (2009).

[18] G. S. Grest and K. Kremer, Physical Review A 33, 3628 (1986).

[19] M. Revenga, I. Zúñiga, and P. Español, Computer Physics Communications 121-122, 309 (1999).

[20] S. Biagi, A mesoscale investigation of the endothelial glycocalyx and its interaction with blood flow, Theses, Università degli studi La Sapienza (Rome) (2016).

[21] S. Weinbaum, J. M. Tarbell, and E. R. Damiano, Annual Review of Biomedical Engineering 9, 121 (2007).

[22] S. T. Milner, T. A. Witten, and M. E. Cates, EPL (Europhysics Letters) 5, 413 (1988).

[23] S. T. Milner, Science 251, 905 (1991).

[24] M. J. A. Hore, J. Ford, K. Ohno, R. J. Composto, and B. Hammouda, Macromolecules 46, 9341 (2013).
[25] E. Currie, M. Wagemaker, M. C. Stuart, and A. Van Well, Physica B: Condensed Matter 283, 17 (2000).

[26] D. I. Dimitrov, A. Milchev, and K. Binder, The Journal of chemical physics 127, 084905 (2007).

[27] K. Binder, Monte Carlo and molecular dynamics simulations in polymer science (Oxford University Press, 1995).

[28] M. Deng, X. Li, H. Liang, B. Caswell, and G. E. Karniadakis, Journal of Fluid Mechanics 711, 192 (2012).

[29] S. Alexander, Journal De Physique 38, 983 (1977).

[30] De Gennes, P.G., J. Phys. France 37, 1445 (1976).

[31] P. G. de Gennes, Macromolecules 13, 1069 (1980).

[32] R. Asaro and W. Tiller, Metallurgical Transactions 3, 1789 (1972).

[33] M. A. Grinfeld, Sov. Phys. Dokl. 31, 831 (1986).

[34] K. Kassner and C. Misbah, Europhysics Letters (EPL) 28, 245 (1994).

[35] A. Onuki, Phys. Rev. A 39, 5932 (1989).

[36] V. Kumaran, G. H. Fredrickson, and P. Pincus, J. Phys. II France 4, 893 (1994).

[37] L. D. Landau and E. M. Lifshitz, Theory of Elasticity, 3rd (Pergamon Press, Oxford, UK, 1986).

[38] M. Dabagh, P. Jalali, P. J. Butler, and J. M. Tarbell, Journal of The Royal Society Interface 11, 20140431 (2014).

[39] Y. Yao, Three-dimensional flow-induced dynamics of the endothelial surface glycocalyx layer, Ph.D. thesis, Massachusetts Institute of Technology (2007).

[40] V. Kumaran and R. Muralikrishnan, Phys. Rev. Lett. 84, 3310 (2000).

[41] Z. Shen, A. Farutin, M. Thiébaud, and C. Misbah, Phys. Rev. Fluids 2, 103101 (2017).

[42] M. Sferrazza, R. A. L. Jones, and D. G. Bucknall, Phys. Rev. E 59, 4434 (1999).

[43] R. D. Groot and P. B. Warren, The Journal of Chemical Physics 107, 4423 (1997).

[44] P. Español and P. B. Warren, Europhysics Letters 30, 191 (1995). 